\title{
Characterizing and Improving Predictive Accuracy in Shock-Turbulent Boundary Layer Interactions Using Data-driven Models
}

\author{
Anand Pratap Singh*, Shaowu Pan ${ }^{\dagger}$, Karthik Duraisamy ${ }^{\ddagger}$ \\ University of Michigan, Ann Arbor, Michigan
}

\begin{abstract}
A data-driven framework is applied to enhance Reynolds-averaged Navier-Stokes (RANS) predictions of flows involving shock-boundary layer interactions. The methodology involves solving inverse problems to infer spatial discrepancies in the Spalart Allmaras (SA) model and projecting these discrepancies to locally non-dimensional flow features using machine learning. The machine-learned reconstruction of the discrepancy is then used within the RANS partial differential equation solver for predictions. The methodology is applied to problems involving transonic flow over an axisymmetric bump, oblique shock-boundary layer interactions and shock train flows. The ability of the model to assimilate data (surface pressure and field velocities) while predicting other quantities (Reynolds stresses) is studied. Different approaches to infer discrepancies are compared, including a form that preserves a log-layer constraint in the SA model.
\end{abstract}

The interaction of shock waves with turbulent boundary layers is an important phenomenon that occurs in many aerospace systems, including high-speed aircraft, missiles, and scramjet engines. Shock-boundary layer interactions (SBLI) have been a topic of extensive study ${ }^{1-3}$ over the past five decades. Reynoldsaveraged Navier-Stokes (RANS) models continue to be indispensable in the modeling of SBLI in practical systems because of the high Reynolds numbers involved. It is well recognized, ${ }^{4}$ however, that RANS models are inaccurate except in the case of weak interactions. This behavior is attributed to the inability of RANS models to respond to a sharp pressure rise and the use of a single turbulent scale in an inherently multiscale environment. Furthermore, post-shock relaxation is also a source of high modeling inaccuracy.

A key deficiency of RANS models as applied to SBLI can be attributed to the fact that they were originally developed and calibrated based on canonical flows that are far removed from the problems of interest. In this work, starting from a baseline RANS model formulation and LES data, we use inverse modeling to characterize the structural form of discrepancies in the model. Based on this information from a number of different SBLI configurations, we intend to modify the functional form of the RANS closure with the eventual goal of obtaining robust and reliable predictive models for complex high-speed flows. This work represents preliminary efforts towards that objective.

The general framework of field inversion and machine learning (FIML) has been developed by the authors over the past three years. ${ }^{5-10}$ The use of machine learning for turbulence modeling has been growing over the past year as exemplified by a number of authors. ${ }^{11-13}$

\section{Field Inversion and Machine Learning (FIML) for Turbulence Modeling}

A schematic of the FIML methodology is shown in Figure 1. Given an existing model, a model correction field ${ }^{a} \boldsymbol{\beta}(\mathbf{x})$ is introduced into the transport equation. Starting with some high-fidelity or experimental data $\mathbf{G}_{d}$, an inverse problem will be formulated to minimize (an appropriately regularized form of) $\left\|\mathbf{G}_{d}-\mathbf{G}_{\boldsymbol{\beta}}\right\|$,

\footnotetext{
*Graduate Student, Aerospace Engineering, University of Michigan, Ann Arbor, AIAA Student Member

${ }^{\dagger}$ Graduate Student, Aerospace Engineering, University of Michigan, Ann Arbor, AIAA Student Member

${ }^{\ddagger}$ Assistant Professor, Aerospace Engineering, University of Michigan, Ann Arbor, AIAA Member

${ }^{\mathrm{a}} \boldsymbol{\beta}$ is a spatial field if the problem is steady and a spatio-temporal field if it is unsteady.
} 
where $\mathbf{G}_{\boldsymbol{\beta}}$ is the predicted output for a given correction field. Note that the intent is not to infer a limited set of parameters that characterizes the correction, but rather to extract the correction field itself. In other words, $\boldsymbol{\beta}$ is sought at every discrete location in the computational domain and inserted into the transport equation. Thus we are directly addressing structural errors and uncertainties in the model.

The correction fields will be obtained for an ensemble of problems (on different data sets $\mathbf{G}_{d}^{1}, \mathbf{G}_{d}^{2} \ldots$, as shown in the figure) representative of the physical phenomena that is to be modeled. Unlike in parameter estimation, in which the inferred parameters can be used directly in simulations, the $\boldsymbol{\beta}(\mathbf{x})$ field by itself is of limited use in quantitative modeling. In other words, having inferred the optimal model correction function $\boldsymbol{\beta}$, it remains to convert the inference into modeling knowledge.

To be useful in predictive modeling, the spatial dependence of $\boldsymbol{\beta}(\mathbf{x})$ has to be transformed into feature space using machine learning. Machine learning effectively constructs a functional form $\boldsymbol{\beta}(\boldsymbol{\eta})$, where $\boldsymbol{\eta}(\mathbf{U}(\boldsymbol{x}))$ are features (derived from flow and turbulence variables $\mathbf{U}$ ) that will be available during the solution process. The elements of the feature vector $\boldsymbol{\eta}$ should preferably be locally non-dimensional quantities ${ }^{\mathrm{b}}$ such that the functional corrections can be translated across different problems.

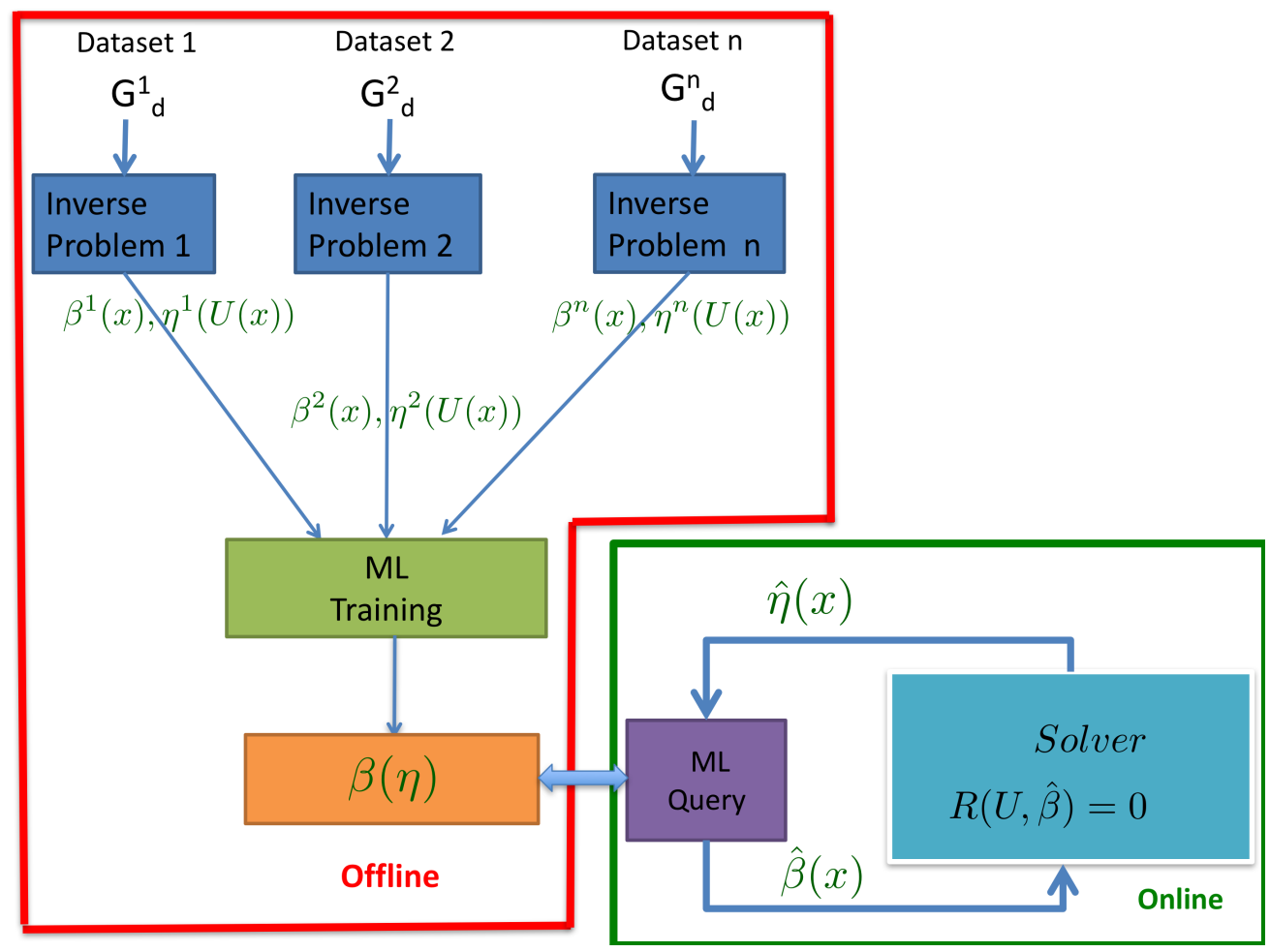

Figure 1. Schematic of field inversion and machine learning framework for data-augmented turbulence modeling

\section{Forward and Inverse Problems}

The governing Reynolds-averaged Navier-Stokes equations are spatially discretized using a cell-centered finite-volume scheme on a polyhedral unstructured mesh. ${ }^{14,15}$ The flow and turbulence model equations are solved in a fully-coupled fashion ${ }^{15}$ using implicit Euler time integration with local time-stepping. To obtain information about the modeling discrepancy, inverse problems are solved. The procedure begins with the introduction of a spatially varying field $\boldsymbol{\beta}(x, y)$ into the model equation. In the case of the Spalart-Allmaras (SA) model below, this term is introduced as a multiplier prefacing the production term.

$$
\frac{D \tilde{\nu}}{D t}=\boldsymbol{\beta}(\mathbf{x}) P(\tilde{\nu}, U)-D(\tilde{\nu}, U)+T(\tilde{\nu}, U)
$$

${ }^{\mathrm{b}}|S| \tau$ is an example of an acceptable feature, where $|S|$ is the magnitude of the strain-rate tensor and $\tau$ is a turbulent time-scale. 
Although the multiplier is introduced to the production term, it is equivalent to adding an additional source term $\delta(x)=(\boldsymbol{\beta}(\mathbf{x})-1) P(\tilde{\nu}, U)$. Effectively, the addition of $\boldsymbol{\beta}(\mathbf{x})$ changes the net balance of all source terms and hence, there is no constraint on the value $\boldsymbol{\beta}(\mathbf{x})$ can take.

In this work, we employ the Bayesian formulation to infer an improved estimate for $\boldsymbol{\beta}(\mathbf{x})$ (and hence the eddy viscosity) within the SA model. The Bayesian approach ${ }^{16}$ provides a formalism to quantify the posterior probability distribution $q(\boldsymbol{\beta} \mid \mathbf{d})$, given a prior distribution $(p(\boldsymbol{\beta}))$, data vector $\mathbf{d}$, and a likelihood function $h(\mathbf{d} \mid \boldsymbol{\beta})$. The Bayes theorem states that

$$
q(\boldsymbol{\beta} \mid d)=\frac{h(\mathbf{d} \mid \boldsymbol{\beta}) p(\boldsymbol{\beta})}{c},
$$

where $c=\int h(\mathbf{d} \mid \boldsymbol{\beta}) p(\boldsymbol{\beta}) d \boldsymbol{\beta}$. In its current form, the solution to Eq. 2 is intractable using a sampling-based method because of the infinite-dimensional nature of $\boldsymbol{\beta}$. The problem is made finite-dimensional by redefining it such that value of $\boldsymbol{\beta}$ is inferred at every point in the computational domain of the RANS grid ${ }^{\mathrm{c}}$.

To promote tractability of the computation of the posterior properties, $q(\boldsymbol{\beta} \mid d) \equiv e^{-\left(\boldsymbol{\beta}-\boldsymbol{\beta}_{\text {prior }}\right)^{T} \mathbf{C}_{\text {prior }}^{-1}\left(\boldsymbol{\beta}-\boldsymbol{\beta}_{\text {prior }}\right)}$ and $h(\mathbf{d} \mid \boldsymbol{\beta}) \equiv e^{-\mathbf{F}^{T} \mathbf{C}_{o b s}^{-1} \mathbf{F}}$ are approximated to be Gaussian. In these expressions, $\mathbf{F}$ is a vector with $i^{\text {th }}$ element $f_{i}=d_{i, R A N S}-d_{i, b e n c h m a r k} ; d_{i}$ is the $i^{\text {th }}$ data point and can be any flow-dependent quantity such as pressure, skin-friction, or velocity. $\mathbf{C}_{o b s}$ and $\mathbf{C}_{\text {prior }}$ are the observational and the prior covariance matrices. $\boldsymbol{\beta}_{\text {prior }}$ is the prior mean of the parameters (and also corresponds to the base model). Under Gaussian assumptions, the maximum a posteriori (MAP) estimate ${ }^{17}$ is taken to be representative of the mean of the distribution. The MAP can be computed by maximizing the numerator in Eq. 2, or equivalently by solving the following deterministic minimization problem:

$$
\boldsymbol{\beta}_{M A P}=\arg \min _{\boldsymbol{\beta}} \mathfrak{J}(\boldsymbol{\beta})=\arg \min _{\boldsymbol{\beta}} \frac{1}{2}\left[\mathbf{F}^{T} \mathbf{C}_{\text {obs }}^{-1} \mathbf{F}+\left(\boldsymbol{\beta}-\boldsymbol{\beta}_{\text {prior }}\right)^{T} \mathbf{C}_{\text {prior }}^{-1}\left(\boldsymbol{\beta}-\boldsymbol{\beta}_{\text {prior }}\right)\right] .
$$

The resulting optimization problem is still high-dimensional, but the posterior distribution is not determined by sampling methods; instead, the covariance is approximated by linearizing about the MAP point. This leads to a definition of the covariance in terms of the inverse of the Hessian of the objective function, $\mathfrak{J}(\boldsymbol{\beta})$,

$$
\mathbf{C}_{\text {posterior }, i, j}=\left.\left[\frac{d^{2} \mathfrak{J}(\boldsymbol{\beta})}{d \beta_{i} d \beta_{j}}\right]^{-1}\right|_{\boldsymbol{\beta}_{M A P}} .
$$

The deterministic minimization problem to obtain the MAP solution is solved using a gradient-based optimization algorithm. The required sensitivities are calculated using an efficient adjoint-based formulation. The framework is described in more detail in Ref. 6 .

\section{Machine Learning}

The spatial functions $\boldsymbol{\beta}(\mathbf{x})$ are projected onto a feature space using a machine learning algorithm. Machine learning models are trained using locally non-dimensional flow features. The set of features that were evaluated includes $\left\{\chi, S / \Omega, \tau / \tau_{\text {wall }}, P / D\right\}$ : the ratio of eddy viscosity to laminar viscosity, the ratio of strain-rate magnitude to vorticity magnitude, the ratio of the Reynolds stress magnitude to the wall shear stress, and the ratio of the production term to the destruction term, respectively. The use of locally nondimensional quantities enables the application of the same trained model to flow conditions not part of the initial training. The machine learning model is used in the the partial differential equation solver alongside the RANS equations.

While we have explored various machine learning algorithms, including Gaussian processes ${ }^{9}$ and neural networks, ${ }^{18}$ we use Adaptive Boosting (Adaboost) in the present work. It was originally developed by Freund et al., ${ }^{19}$ though we use the enhanced version by Drucker ${ }^{20}$ implemented in the Python package scikit-learn. ${ }^{21}$ The basic concept of Adaboost is to iteratively apply weak learners to focus on the data that previous learners failed to learn. For each iteration, point that are mistakenly predicted by the current learners are marked with higher weights, so that the next iteration perceives them as being more important.

Given training data $\left(\eta_{i}, \boldsymbol{\beta}_{i}\right)_{i=1, N}$, the general steps in Adaboost regression are as follows: ${ }^{20}$

${ }^{\mathrm{c}}$ Note that is still a very high-dimensional problem. 
1. Assign an initial weight, $w_{i}=1 / N$, to each data point.

2. Choose $N$ samples from the data (with replacement). Probability of choosing a data point is based on its weight.

3. Construct a predictor (e.g., decision tree regressor with a maximum depth and number of features) that minimize the error on current sample of the weighted training data.

4. Compute a measurement of the confidence of the predictor in step 3 based on a chosen loss function.

5. Update the weights $w_{i}$ based on the confidence and point-wise loss function.

6. Repeat from Step 2 until the desired total number of predictors is reached.

7. The regression result is computed as a weighted median of all predecessor predictors based on the confidence of each predictor and learning rate.

\section{Axisymmetric Transonic Bump}

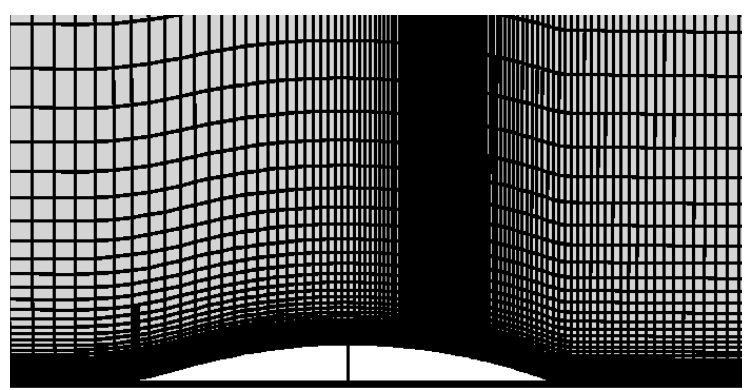

(a) Mesh

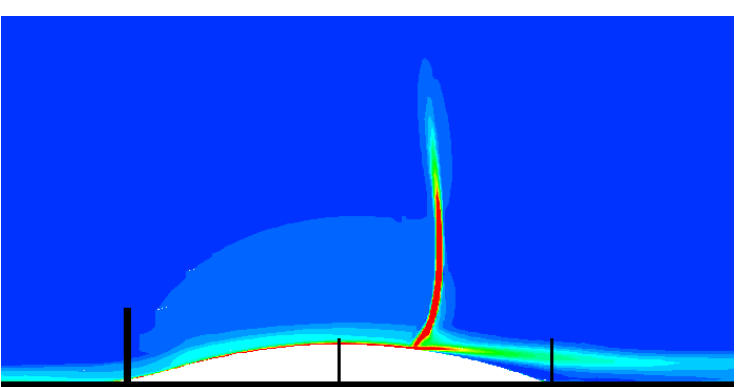

(b) Density gradient

Figure 2. Mesh and density graident contours for the axisymmetric transonic bump.

The Bachalo-Johnson axisymmetric transonic bump ${ }^{22}$ is a standard validation case for turbulence models. The presence of an adverse pressure gradient, a shock, and the resulting region of separation makes it a particularly challenging test case for RANS models. The flow setup consists of an axisymmetric cylinder with a bump of length 1 , and a uniform freestream with a Mach number of 0.875 and Reynolds number of 2.76 Million. A mesh of size $181 \times 81$ is used (Fig. 2a). The computational domain ahead of the bump, is extended to ensure a sufficiently thick boundary layer at the bump onset. Axisymmetric RANS equations are solved with no-slip boundary conditions at the lower wall and characteristic boundary conditions at the other three boundaries. Fig. 2b shows the density gradient using the base Spalart-Allmaras (SA) model, highlighting the shock structure.

RANS-based models are known to predict inaccurate boundary-layer separation and SBLI. This deficiency results in a more aft shock location and a larger than expected separation region, which are apparent in Fig. 3 , a comparison of the wall pressure and skin friction between the base (SA) model and direct numerical simulation (DNS). These deficiencies can be reduced by solving the inverse problem, as shown in the next section.

\section{A. Inverse solutions}

For this flow, we solve two inverse problems: one using wall pressure, a surface quantity, as data, and the other using the full velocity vector field as data. The data came from a DNS by Spalart et al. ${ }^{23}$ The purpose of this is to compare the inverse solution using sparse data with that using full field data as, for a practical problem, we rarely have access to the latter. Fig. 3 shows the wall pressure and the skin friction for the two cases compared with the base SA model and DNS. The surface quantities for both inversions show good agreement with DNS. Inversion based on pressure performs slightly better than inversion based on velocity; this is not entirely surprising as the inverse solution is explicitly driven by a surface quantity. Reynolds shear 


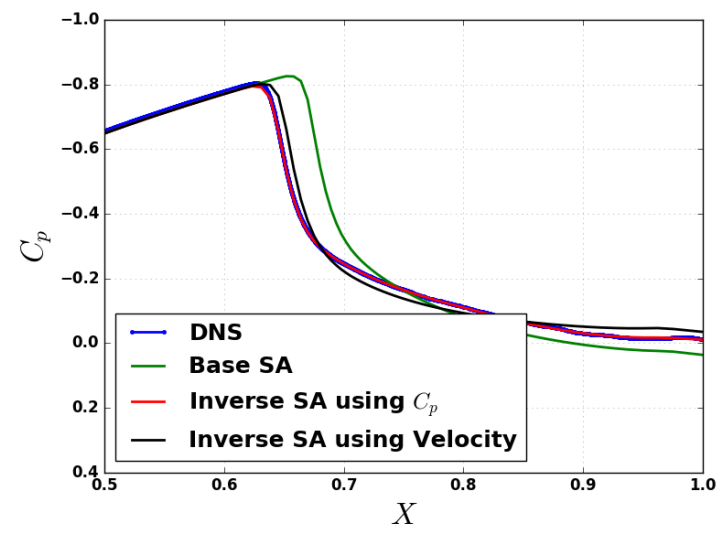

(a) $C_{P}$

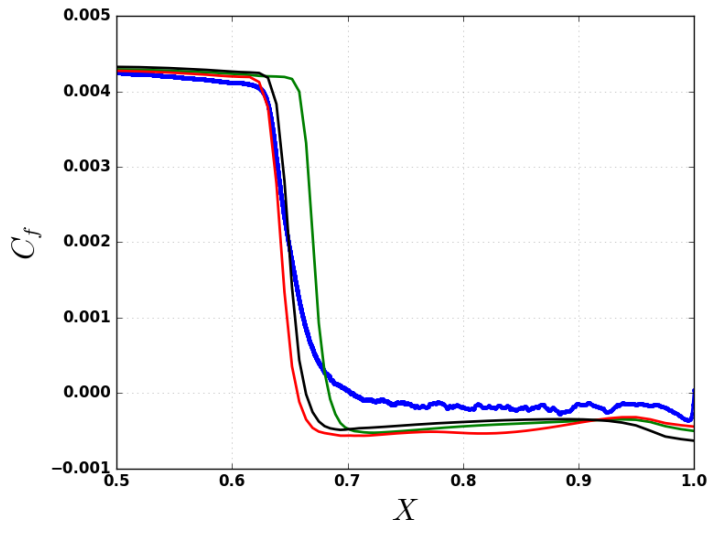

(b) $C_{f}$

Figure 3. Wall pressure and skin friction using DNS, base SA and inverse SA models using pressure and velocity based objective functions.

stress profiles at different axial locations are shown in Fig. 4. Overall, there is an improvement in Reynolds stress predictions. Furthermore, there is no conclusive trend to suggest that velocity-based inversion is better than inversion based on the wall pressure. While it is not prudent to draw general conclusions, in the absence of full fields of data (as will be the case in high Reynolds number flows), it is not unreasonable to use surface quantities for inversion. In fact, the authors have demonstrated that for separated flows over airfoils, the lift coefficient is sufficient to obtain an acceptable $\boldsymbol{\beta}(\mathbf{x})$ field. ${ }^{18}$

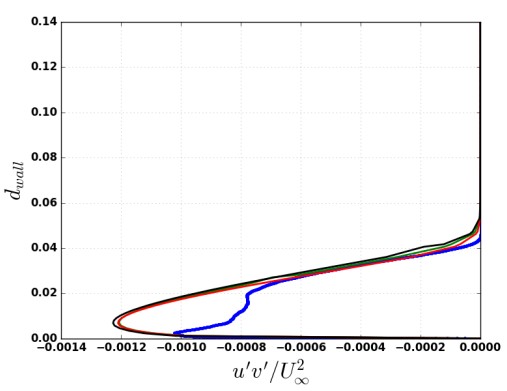

(a) $X=-0.25$

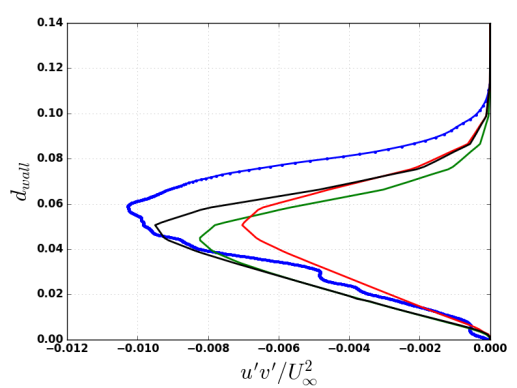

(b) $X=1.0$

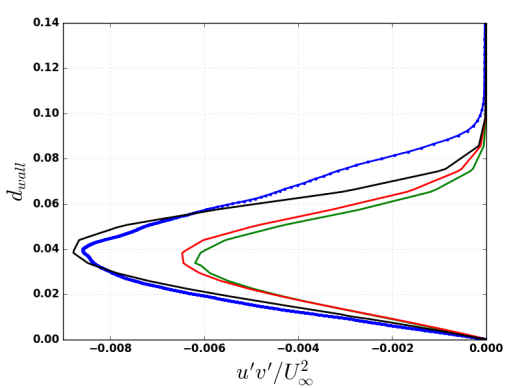

(c) $X=1.25$

Figure 4. Reynolds shear stress using DNS, base SA and inverse SA models using pressure and velocity based objective functions.

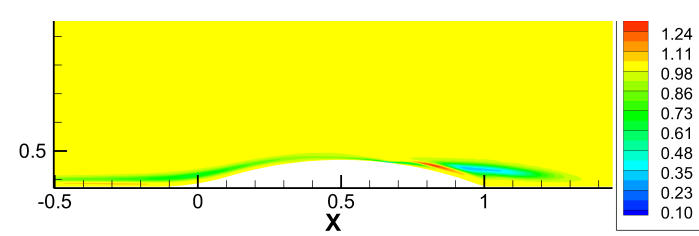

(a) Using wall pressure

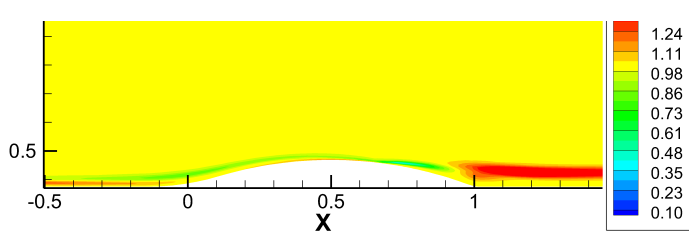

(b) Using velocity field

Figure 5. Inverse solution $\beta(x)$ using pressure based and velocity based objective functions.

Fig. 5 shows that for the two inverse solutions, the $\boldsymbol{\beta}(\mathbf{x})$ fields are considerably different after the point of separation, but they share a high degree of similarity in the attached region. This points towards a universal trend in the correction term and suggests that the solution fields, at least in the attached region, are not a result of overfitting. 


\section{A constrained approach to modifying the SA model}

In the previous section, we modified the SA model by introducing a multiplier to the production term. In the present structure, there are no constraints on the values $\boldsymbol{\beta}(\mathbf{x})$ can assume. It is desirable for corrections to the base model to maintain accuracy in situations in which the base model is known to perform well. To achieve this, one might constrain $\boldsymbol{\beta}(\mathbf{x})$, or modify the model at a lower level to respect physics-based constraints.

In this section, we modify the dissipation term in the SA model by modifying the function $f_{w}$. The dissipation term in the SA model is given by,

$$
D=c_{w 1} f_{w}\left[\frac{\tilde{\nu}}{d}\right]^{2}
$$

The function $f_{w}$ is defined as:

$$
f_{w}=g\left[\frac{1+c_{w 3}^{6}}{g^{6}+c_{w 3}^{6}}\right]^{\frac{1}{6}}, \quad g(r)=r+c_{w 2}\left(r^{6}-r\right), \quad r=\frac{\tilde{\nu}}{\tilde{\Omega} \kappa^{2} d^{2}},
$$

definition of all the symbols and values of different constants can be found in Spalart et al. ${ }^{24}$ The function $g(r)$, and hence $f_{w}$, is constrained to be unity in the log layer i.e. $g(1)=1$. Following this, $c_{w 2}$ is calibrated so as to match skin friction for a flat-plate boundary layer. We modify this function by introducing a spatial correction function, $\boldsymbol{\beta}(\mathbf{x})$, as follows,

$$
g(r)=r+\boldsymbol{\beta}(\mathbf{x}) c_{w 2}\left(r^{6}-r\right) .
$$

The log layer constraint is still satisfied, and we can infer $\boldsymbol{\beta}(\mathbf{x})$ as in the previous section. We perform the inversion using wall pressure data. Fig. 6 shows the resulting wall pressure and skin friction coefficient. The solution is in good agreement with DNS and the new modification does not restrict the inference. Fig. 9a shows the resulting contour of $\boldsymbol{\beta}(\mathbf{x})$ and Fig. 7 shows the plot for $f_{w}(r)$ vs $r$. The baseline SA model dictates $f_{w}(1)=1$ and $\frac{d f_{w}(1)}{d r}=1$, which are retained.

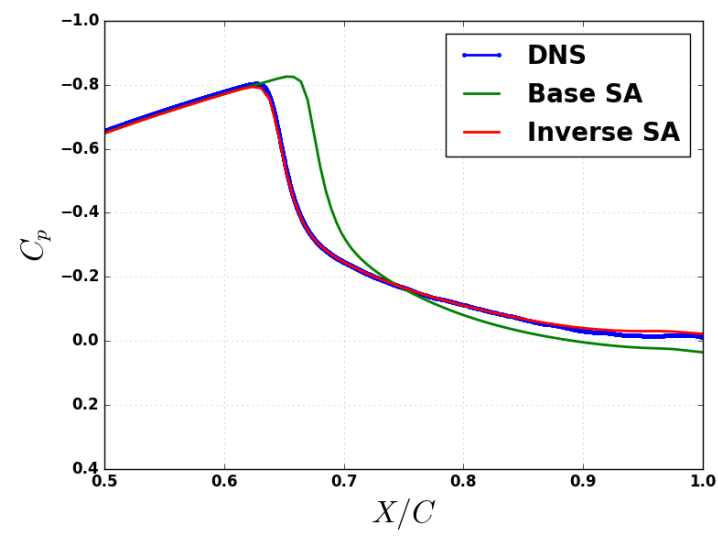

(a) $C_{P}$

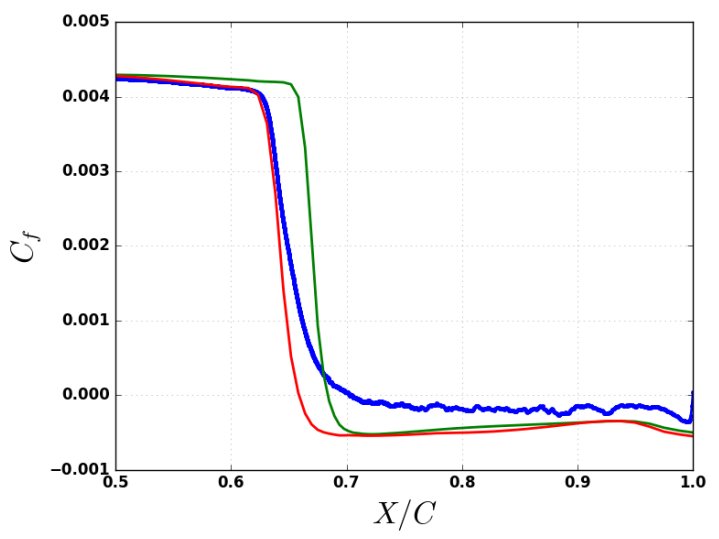

(b) $C_{f}$

Figure 6. Wall pressure and skin friction for DNS, base SA model and inverse SA model by modifying $f_{w}$.

\section{B. Reconstruction using Adaboost}

The spatially varying inverse solution, $\boldsymbol{\beta}(\mathbf{x})$, is projected onto the feature space using Adaboost (as in Section III) to obtain $\boldsymbol{\beta}(\eta)$. Values of five-fold cross-validation coefficients of determination $\left(R^{2}\right)$ are listed in Table 1. As seen in the table, the trained model is robust with respect to available data. Fig. 8 shows the inverse solution and the machine-learned solution for $\boldsymbol{\beta}(\eta)$ as a multiplier of the production term, and Fig. 9 shows $\boldsymbol{\beta}(\eta)$ as an element of $f_{w}$. The machine-learned reconstruction is an accurate representation of the inverse solution. Fig. 7 compares the machine learned function $f_{w}$ with the base and the inverse $f_{w}$. The wall 


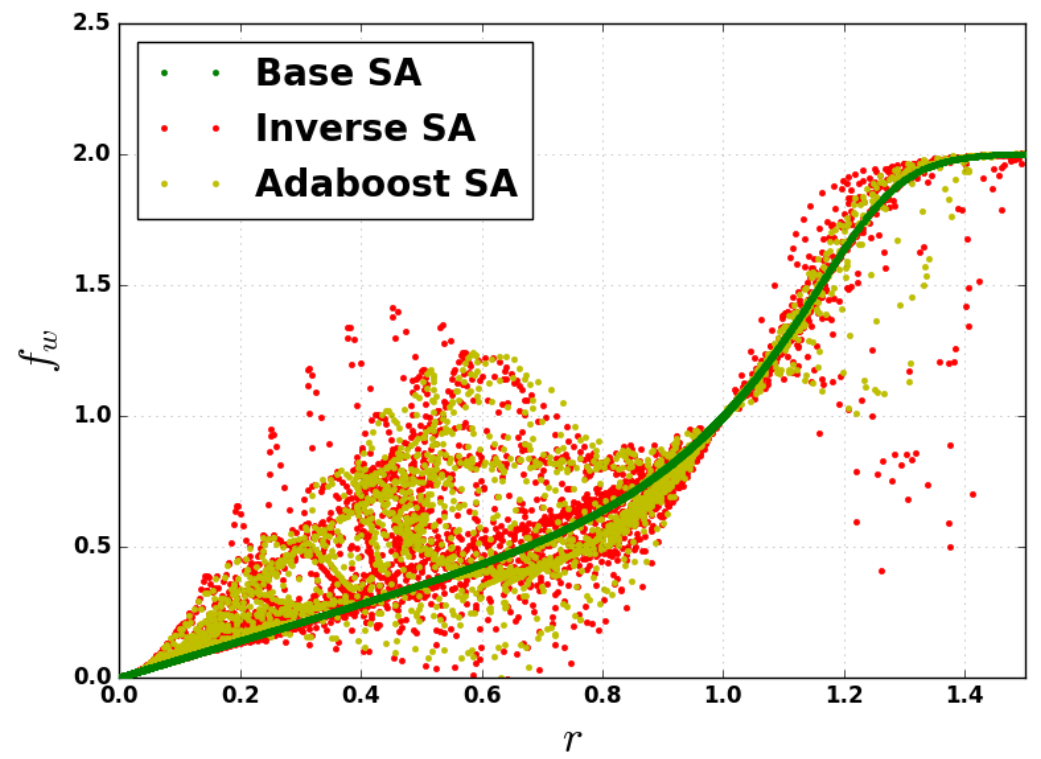

Figure 7. $f_{w}$ vs $r$ for the base SA, inverse SA and the machine learning augmented SA model.

\begin{tabular}{lll} 
CV number & $R_{\text {train }}^{2}$ & $R_{\text {validation }}^{2}$ \\
\hline 1 & 0.9958 & 0.9579 \\
2 & 0.9957 & 0.9655 \\
3 & 0.9961 & 0.9454 \\
4 & 0.9956 & 0.9509 \\
5 & 0.9957 & 0.9387
\end{tabular}

Table 1. Cross validation of Adaboost for transonic axisymmetric bump production multiplier.

pressure and the skin friction computed with the machine-learned corrections as part of the RANS solver are shown in Fig. 10. There is a good agreement between the solution obtained using the machine learning augmented model and DNS.

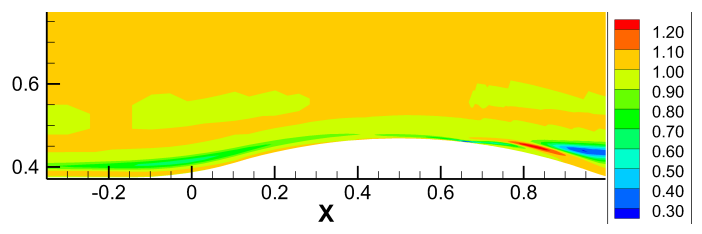

(a) Inverse $\boldsymbol{\beta}(\mathbf{x})$

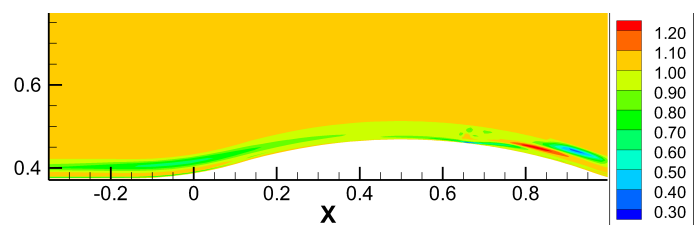

(b) Machine learned $\boldsymbol{\beta}^{\star}(\eta)$

Figure 8. Comparison of the inverse solution and machine learning-based reconstruction of inverse solution using modification based on production term. 


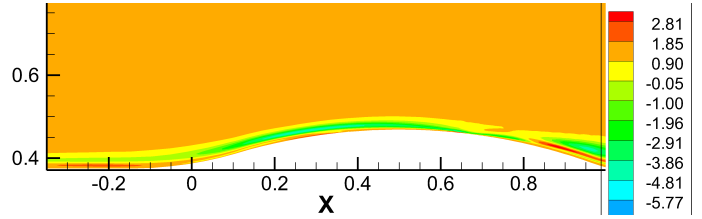

(a) Inverse $\boldsymbol{\beta}(\mathbf{x})$

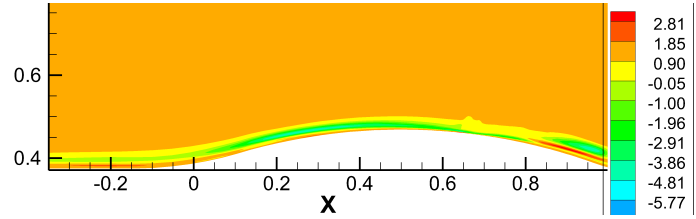

(b) Machine learned $\boldsymbol{\beta}(\eta)$

Figure 9. Comparison of the inverse solution and machine learning-based reconstruction of inverse solution using modification based on $f_{w}$.

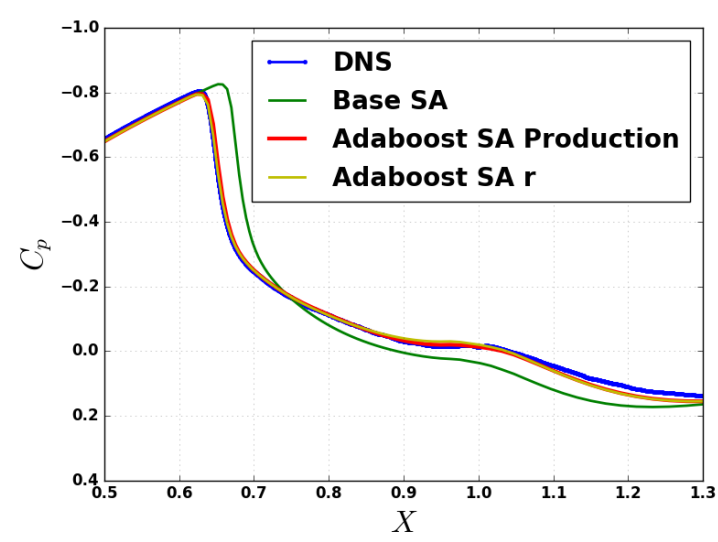

(a) Wall pressure

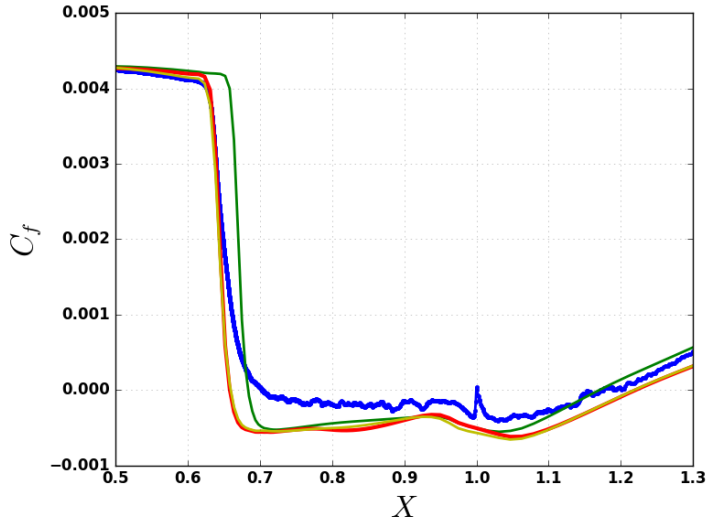

(b) Skin friction

Figure 10. Pressure and skin friction from DNS, base SA model, machine learning augmented model-based on production and $f_{w}$ modifications. 


\section{Oblique Shock-Boundary Layer Interactions}

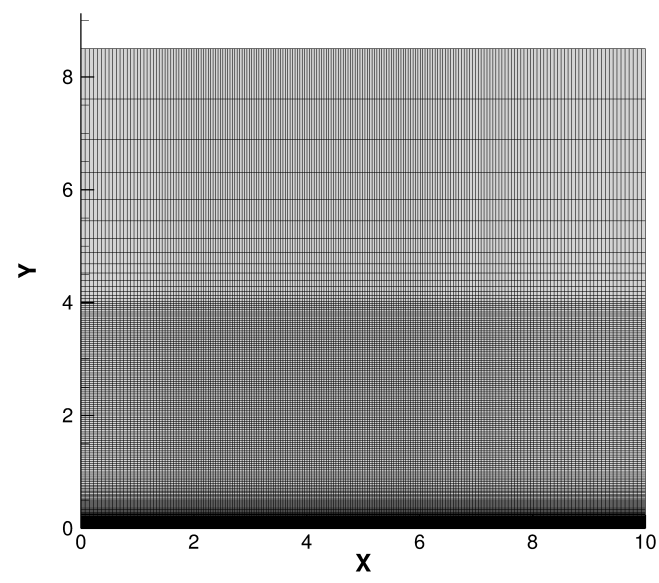

(a) Mesh

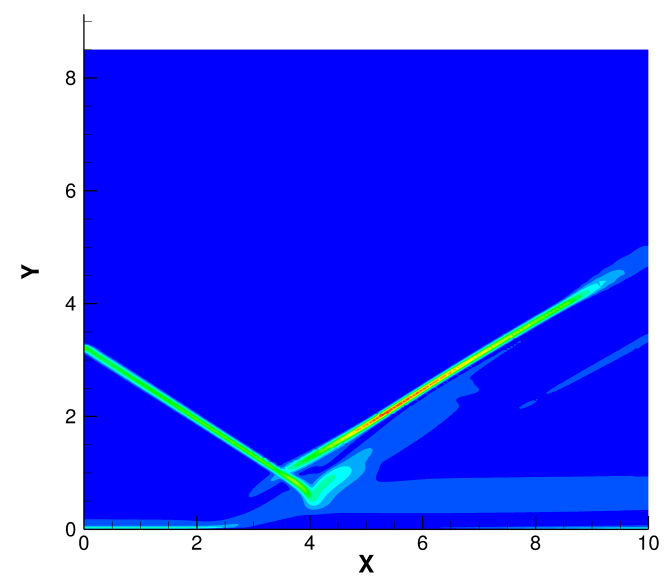

(b) Density gradient

Figure 11. Mesh and density gradient contours for shock-boundary layer interaction setup.

To further examine the generality of this methodology, simulations of the interaction of a single oblique shock with a turbulent boundary layer were considered. The inverse solution was obtained by the authors in a prior work ${ }^{7}$ using the $k-\omega$ model. The flow conditions attempt to emulate the benchmark setup in Morgan et al., ${ }^{25}$ which employs a well-resolved large-eddy simulation (LES). The incoming boundary layer has a free-stream Mach number of 2.28, an inlet momentum-thickness Reynolds number of 1500 , and a thickness of 0.87 grid units. A grid of extent 10 units in the streamwise direction and 15 units in the wall-normal direction is discretized using a Cartesian mesh of 201 uniform points in the streamwise direction and 151 points in the wall-normal direction. The undisturbed shock is aligned at an angle of $8^{\circ}$ to the freestream and its reflection point is at the center point of the wall domain. The inlet boundary layer is generated using a precursor flat plate simulation. Rankine-Hugoniot conditions are used to obtain the shock jump conditions, which are then enforced at the inlet plane.

\section{A. Inverse solution}

Similar to the transonic bump, a correction function $\boldsymbol{\beta}(x, y)$ is multiplied to the production term of the $\mathrm{SA}$ equation. The coefficient of pressure $\left(C_{p}\right)$ at the lower wall from the LES solution ${ }^{25}$ is used as the benchmark data for inversion. Fig. 12 compares the pressure and friction coefficients using the base SA model, the inverse SA model, and the LES. The posterior $C_{p}$ tracks the LES consistently. As evident in the $C_{f}$ plot, the location and length of the separation bubble is more accurately predicted by the inverse model. Fig. 13a shows the contour of the inverse solution, $\boldsymbol{\beta}(\mathbf{x})$, suggesting a significant model discrepancy in the interaction region.

\section{B. Reconstruction using Adaboost}

Adaboost is again used to reconstruct the spatial inverse solution, $\boldsymbol{\beta}(\mathbf{x})$, in terms of the flow features. Cross-validation $R^{2}$ values are shown in Table 2. Fig. 13b shows the reconstructed function $\boldsymbol{\beta}^{\star}(\eta)$, a good approximation to $\boldsymbol{\beta}(\mathbf{x})$. The RANS equations are solved using this machine learning augmented correction term and the resulting wall quantities are shown in Fig. 14. The solution using the machine learning augmented model is in good agreement with LES and is a significant improvement over the base SA model. 


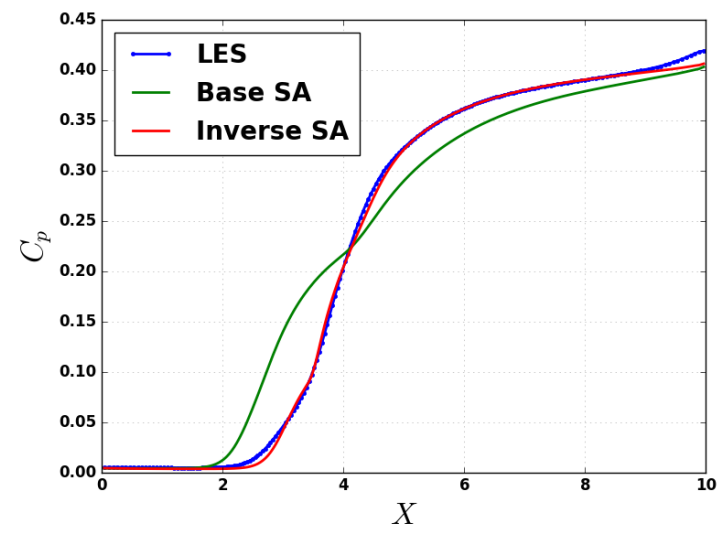

(a) $C_{p}$

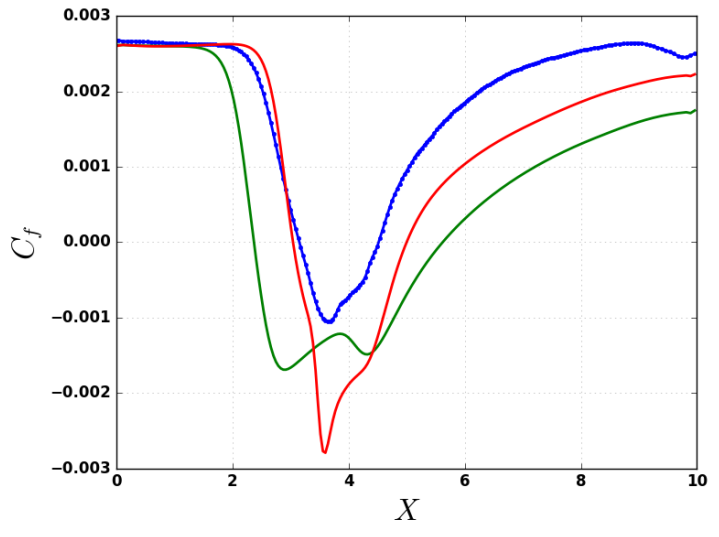

(b) $C_{f}$

Figure 12. Pressure and friction coefficient for the LES, base SA and the Inverse SA model.

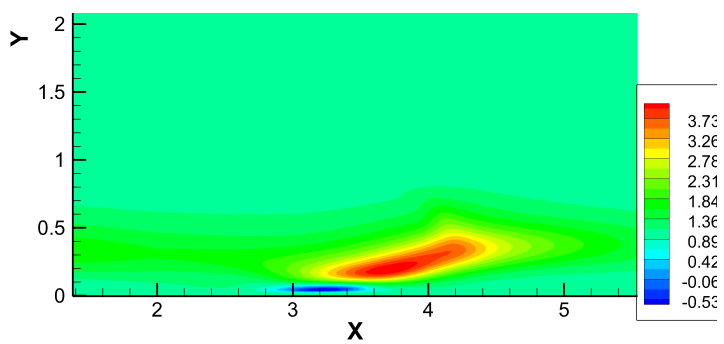

(a) Inverse $\boldsymbol{\beta}(\mathbf{x})$

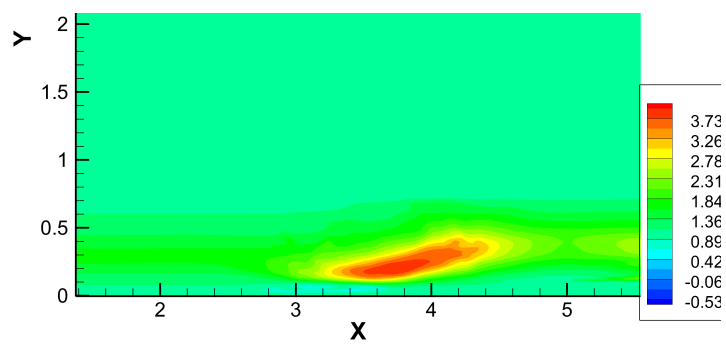

(b) Machine Learned $\boldsymbol{\beta}^{\star}(\eta)$

Figure 13. Inverse solution $\beta(x)$ and machine learning constructed $\boldsymbol{\beta}^{\star}(\eta)$.

\section{Application to isolator shock train}

The shock-train configurations corresponds to the LES of a constant area isolator performed by Morgan et al. ${ }^{26}$ The cross-sectional dimension of the duct is $33.75 \mathrm{~mm}$ (height) by $76.2 \mathrm{~mm}$ (width). At the inlet, the Mach number of the core flow is 1.61 and the momentum-thickness Reynolds number is $R e_{\theta} \approx 1660$, resulting in a confinement ratio of 0.32 . The length of the duct is $400 \mathrm{~mm}$. The outlet to inlet pressure ratio is set to 2.23085. These numbers correspond to the experimental studies of Caroll and Dutton, ${ }^{27}$ but at a reduced Reynolds number. Figure 15 qualitatively compares the LES and experiments. Detailed validation studies are presented in Ref. 26 using using the Spalart-Allmaras, Menter SST, and Wilcox $k-\omega$ models. The solutions obtained from RANS simulations were seen to vary dramatically depending on the turbulence model. The location of the initial shock, given the same back pressure for each model was shown to be offset by nearly 12 inlet boundary layer thickness compared to the LES.

For the rest of the study, the Spalart Allmaras (SA) model is considered for improvement.

\begin{tabular}{lll} 
CV number & $R_{\text {train }}^{2}$ & $R_{\text {validation }}^{2}$ \\
\hline 1 & 0.9931 & 0.9015 \\
2 & 0.9939 & 0.8795 \\
3 & 0.9940 & 0.9078 \\
4 & 0.9937 & 0.9238 \\
5 & 0.9937 & 0.9067
\end{tabular}

Table 2. Cross validation of Adaboost for oblique shock-boundary layer interaction. 


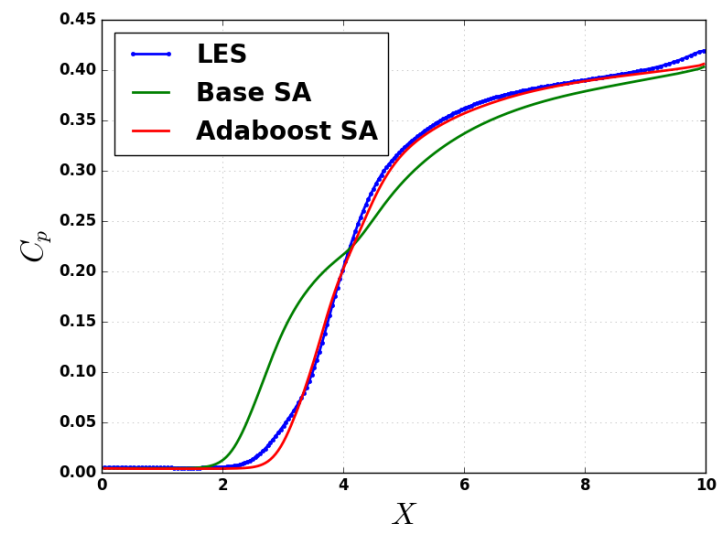

(a) $C_{p}$

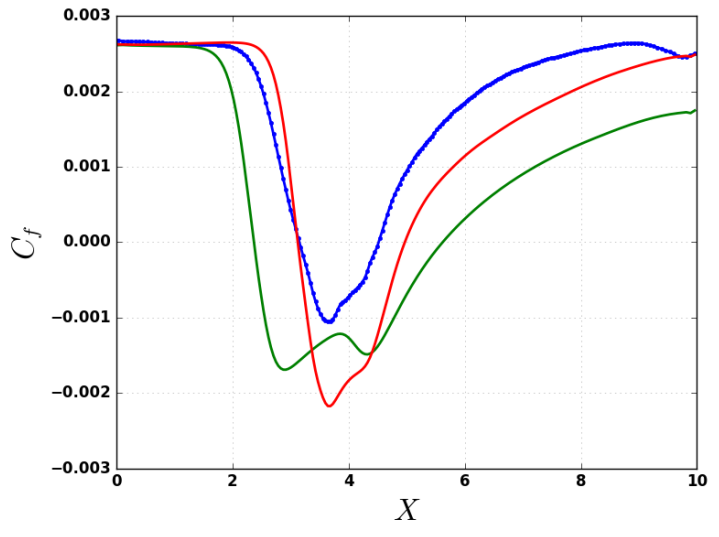

(b) $C_{f}$

Figure 14. Pressure and friction coefficient for the LES, base SA and the ML augmented SA model.
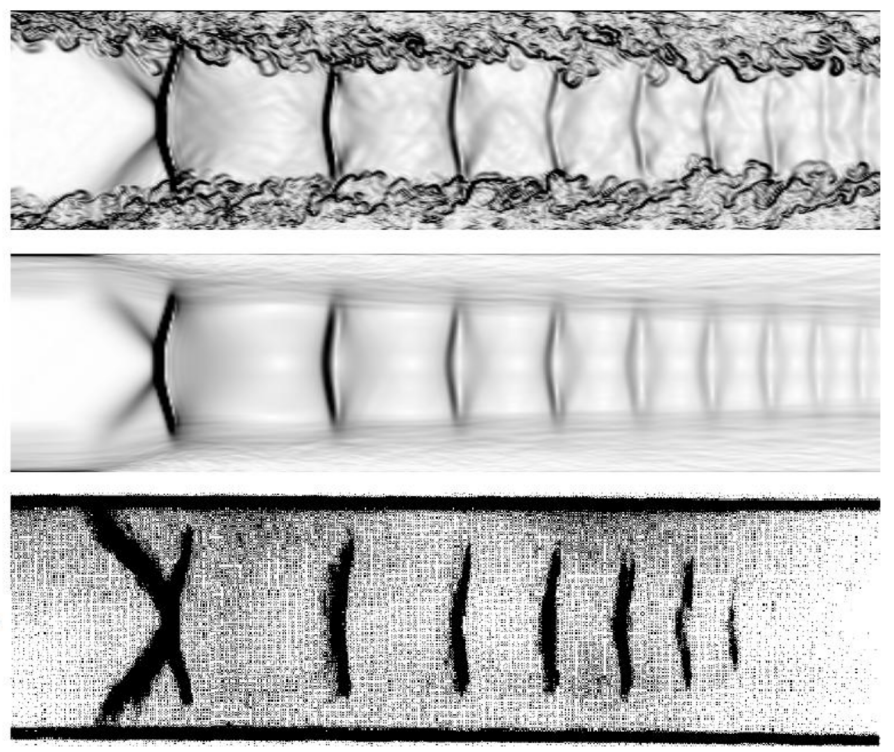

Figure 15. Shock train in a constant area isolator: (Top) LES contours of instantaneous $|\nabla \rho|$. (Middle) LES-C contours of time- and span- averaged $|\nabla \rho|$. (Bottom) Experimental schlieren image. ${ }^{27}$

For the inversion, wall pressure and skin-friction from the LES is used as data. Figure 16 shows the LES, base SA and the inverse SA solutions. Quite clearly, the inverse model is able to assimilate the data successfully. By just using the wall data, inverse SA model leads to much-improved pressure at the centerline. Further, as seen from Figure 17, the solution is improved in the entire flow field, lending further credibility to the inverse solution.

Figure 18(a) shows the contours of the correction function $\boldsymbol{\beta}(\mathbf{x})$. The correction function is higher than unity in the shock boundary layer interaction region and less than unity around the sonic line. There are some qualitative similarities with regard to the region of high $\boldsymbol{\beta}$ at the foot of the shock, offering promise for the development of a universal correction.

The machine-learned reconstruction of the correction function is shown in Figure 18(b). For this case, the quality of reconstruction is not as good as in previous cases, mainly because the discrepancy in the baseline model is very large. Due to this deficient reconstruction, the first shock location using the ML augmented model is about 5 boundary layer thicknesses downstream in comparison to the LES (or the inverse) solution. However, there is still a significant improvement over the base SA model, and the error in the shock location is reduced by approximately two-third. The resulting pressure distributions are shown in Figure 16. 


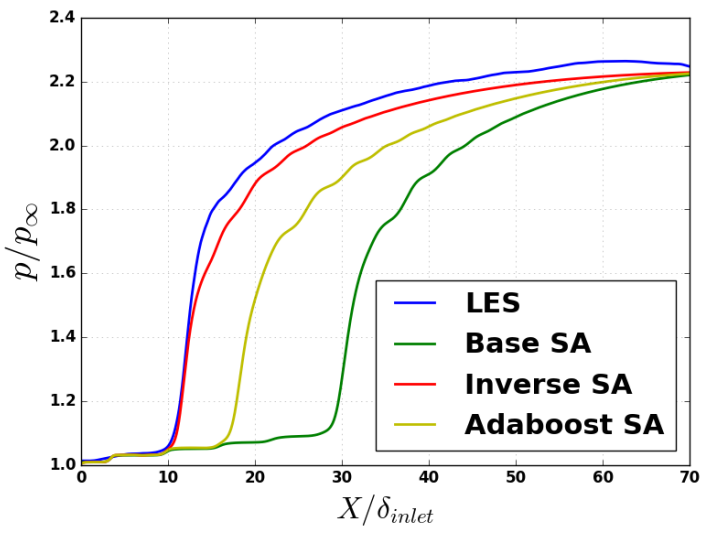

(a) Wall

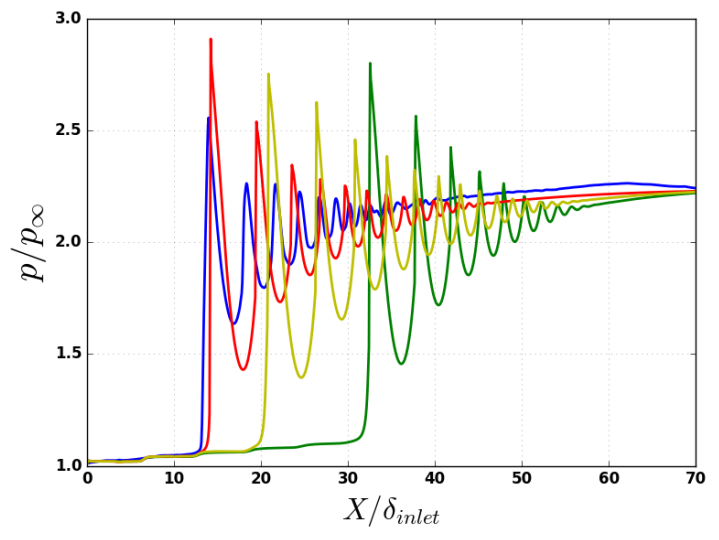

(b) Centerline

Figure 16. Pressure for the LES, base SA, inverse SA and the machine learning augmented SA model.

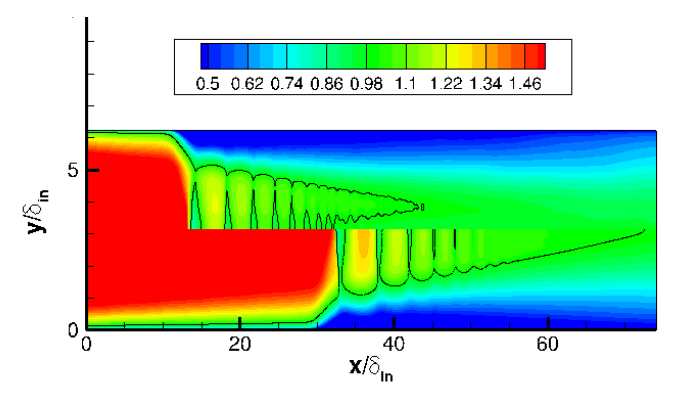

(a) Base SA (bottom) vs LES (top)

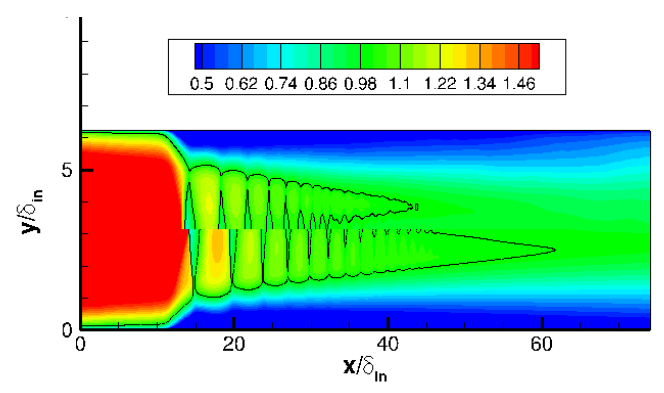

(b) Inverse SA (bottom) vs LES (top)

Figure 17. Mach number predictions (lines represent sonic conditions) 


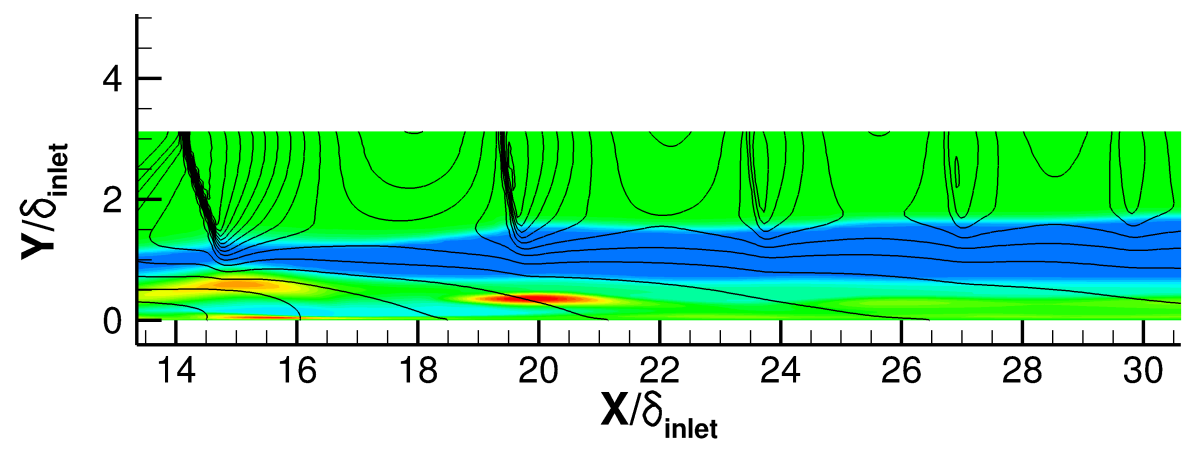

(a) Inverse $\boldsymbol{\beta}(\mathbf{x})$

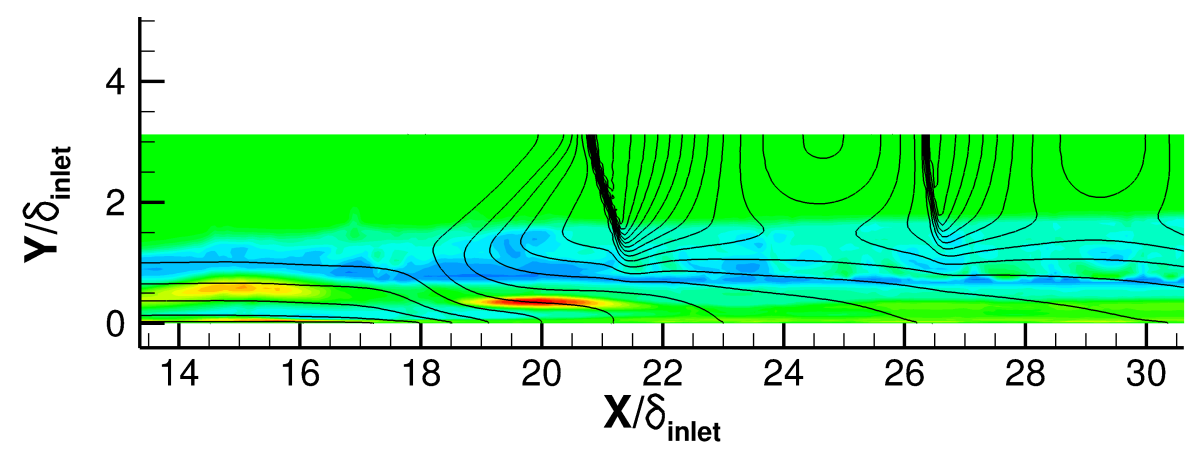

(b) Machine Learned $\boldsymbol{\beta}^{\star}(\eta)$

Figure 18. Density contours using the inverse solution and its machine learned reconstruction, colored by $\beta$.

\section{Summary}

A data-driven framework comprising of full-field inversion and machine learning was used to augment the Spalart-Allmaras (SA) model for flows involving the interaction of shocks with turbulent boundary layers. The methodology was applied to problems involving transonic flow over an axisymmetric bump, oblique shock-boundary layer interaction and a shock train. Solutions of full-field inversion using wall pressure and the full velocity field were evaluated, and their performance were found to be comparable. An alternate approach of modifying SA model, which satisfies a log-layer constraint, is considered. The constraint does not inhibit the efficacy of full-field inversion.

The full-field inverse solutions were reconstructed in terms of locally non-dimensional features using a machine learning algorithm, Adaboost. The reconstructed fields were embedded into a RANS solver to augment the solution of partial differential equations. The solution with this machine learning augmented model shows significant improvement over the baseline SA model for the transonic bump and the flow involving oblique shock boundary interaction. For the shock train, the machine learning reconstruction is is seen to offer an improvement over the baseline model, but is inferior to the inverse solution. The poor reconstruction can either be due to imperfect training, or our choice of flow features used for training; this remains under investigation. This paper presents preliminary progress towards the development of improved predictive models for turbulent high-speed flow applications.

\section{Acknowledgement}

This work was funded by the NASA Aeronautics Research Institute (NARI) under the Leading Edge Aeronautics Research for NASA program (Monitors: Koushik Datta and Gary Coleman). DNS data was provided by P.R. Spalart and M. Strelets. 


\section{References}

${ }^{1}$ Dolling, D. S. and Murphy, M. T., "Unsteadiness of the Separation Shock Wave Structure in a Supersonic Compression Ramp Flowfield," AIAA Journal, Vol. 21, No. 12, Dec 1983, pp. 1628-1634.

${ }^{2}$ Dolling, D. S., "Fifty Years of Shock-Wave/Boundary-Layer Interaction Research: What Next?" AIAA Journal, Vol. 39, No. 8, Aug 2001, pp. 1517-1531.

${ }^{3}$ Delery, J., Marvin, J. G., and Reshotko, E., "Shock-wave boundary layer interactions," Tech. rep., NATO AGARD, 1986.

${ }^{4}$ Knight, D. D. and Degrez, G., "Shock wave boundary layer interactions in high Mach number flows: A critical survey of current CFD prediction capabilities," Hypersonic experimental and computational capability, improvement and validation, 1998.

${ }^{5}$ Duraisamy, K., Zhang, Z. J., and Singh, A. P., "New Approaches in Turbulence and Transition Modeling Using Datadriven Techniques," AIAA SciTech, Jan 2015.

${ }^{6}$ Parish, E. J. and Duraisamy, K., "A paradigm for data-driven predictive modeling using field inversion and machine learning," J. Comput. Physics, Vol. 305, 2016, pp. 758-774.

${ }^{7}$ Singh, A. P. and Duraisamy, K., "Using field inversion to quantify functional errors in turbulence closures," Physics of Fluids, Vol. 28, No. 4, 2016, pp. 045110.

${ }^{8}$ Parish, E. and Duraisamy, K., "Quantification of turbulence modeling uncertainties using full field inversion," 22nd AIAA Computational Fluid Dynamics Conference, AIAA Aviation, (AIAA 2015-2459), Dallas, TX, Jun 2015.

${ }^{9}$ Zhang, Z. J. and Duraisamy, K., "Machine Learning Methods for Data-Driven Turbulence Modeling," 22nd AIAA Computational Fluid Dynamics Conference, AIAA Aviation, (AIAA 2015-2460), Dallas, TX, Jun 2015.

${ }^{10}$ Duraisamy, K., Zhang, Z. J., and Singh, A. P., "Augmentation of Turbulence Models Using Field Inversion and Machine Learning," AIAA SciTech, Jan 2017.

${ }^{11}$ Ling, J., Kurzawski, A., and Templeton, J., "Reynolds averaged turbulence modelling using deep neural networks with embedded invariance," Journal of Fluid Mechanics, Vol. 807, 2016, pp. 155-166.

${ }^{12}$ Wang, J.-X., Wu, J.-L., and Xiao, H., "Physics-Informed Machine Learning for Predictive Turbulence Modeling: Using Data to Improve RANS Modeled Reynolds Stresses," arXiv preprint arXiv:1606.07987, 2016.

${ }^{13}$ Weatheritt, J., The development of data driven approaches to further turbulence closures, Ph.D. thesis, University of Southampton, 2015.

${ }^{14}$ Pecnik, R., Terrapon, V., Ham, F., and Iaccarino, G., "Full system scramjet simulation," Annual Research Briefs, 2009, pp. 33-45.

${ }^{15}$ Duraisamy, K. and Alonso, J., "Adjoint based techniques for uncertainty quantification in turbulent flows with combustion," 42nd AIAA Fluid Dynamics Conference and Exhibit, 2012, p. 2711.

${ }^{16}$ Stuart, A. M., "Inverse problems: a Bayesian perspective," Acta Numerica, Vol. 19, 2010, pp. 451-559.

${ }^{17}$ Aster, R. C., Borchers, B., and Thurber, C. H., Parameter estimation and inverse problems, Academic Press, 2013.

${ }^{18}$ Singh, A., Medida, S., and Duraisamy, K., "Machine Learning-augmented Predictive Modeling of Turbulent Separated Flows over Airfoils," arXiv preprint arXiv:1608.03990v3, 2016.

${ }^{19}$ Freund, Y. and Schapire, R. E., "A desicion-theoretic generalization of on-line learning and an application to boosting," European conference on computational learning theory, 1995, pp. 23-37.

${ }^{20}$ Drucker, H., "Improving regressors using boosting techniques," ICML, Vol. 97, 1997, pp. 107-115.

${ }^{21}$ Pedregosa, F., Varoquaux, G., Gramfort, A., Michel, V., Thirion, B., Grisel, O., Blondel, M., Prettenhofer, P., Weiss, R., Dubourg, V., et al., "Scikit-learn: Machine learning in Python," Journal of Machine Learning Research, Vol. 12, No. Oct, 2011, pp. 2825-2830.

${ }^{22}$ Bachalo, W. and Johnson, D., "Transonic, turbulent boundary-layer separation generated on an axisymmetric flow model," AIAA journal, Vol. 24, No. 3, 1986, pp. 437-443.

${ }^{23}$ Spalart, P. and Strelets, M., Personal communication.

${ }^{24}$ Spalart, P. and Allmaras, S., "A one-equation turbulence model for aerodynamic flows," 30th Aerospace Sciences Meeting and Exhibit Reno, NV, Jan 1992.

${ }^{25}$ Morgan, B., Duraisamy, K., Nguyen, N., Kawai, S., and Lele, S., "Flow physics and RANS modelling of oblique shock/turbulent boundary layer interaction," Journal of Fluid Mechanics, Vol. 729, 2013, pp. 231-284.

${ }^{26}$ Morgan, B., Duraisamy, K., and Lele, S. K., "Large-eddy simulations of a normal shock train in a constant-area isolator," AIAA journal, Vol. 52, No. 3, 2014, pp. 539-558.

${ }^{27}$ Carroll, B. F. and Dutton, J. C., "Characteristics of multiple shock wave/turbulent boundary-layer interactions in rectangular ducts," Journal of Propulsion and Power, Vol. 6, No. 2, 1990, pp. 186-193. 